\title{
Befunde zur spätglazialen fluvial-limnischen Morphodynamik in kleinen Talungen Vorpommerns
}

\author{
Henrik Helbig \& Pim de KLerk*)
}

Helbig, H. \& DE KLerk, P. (2002): Befunde zur spätglazialen fluvial-limnischen Morphodynamik in kleinen Talungen Vorpommerns. - Eiszeitalter und Gegenwart, 51: 51-66; Hannover 2002.

Keywords: Spätglazial, Dryas-III, Alleröd, fluvial-limnischen, Mecklenburger Stadium

Kurzfassung: Die hier vorgestellten Befunde resultieren aus Untersuchungen in einem Erdgasleitungsgraben, der nördlich der Pommerschen Hauptrandlage über die jungen Grundmoränenplatten des Mecklenburger Stadiums verlief.

Während des Alleröds versumpfte und durch Torfbildung geprägte Talabschnitte und Becken wurden während der Dryas-III vorwiegend mit minerogenen Sedimenten (Sand, zumeist Feinsand, nicht selten mit deutlichem Schluffanteil) verfüllt. Die Dryas-III ist im Vergleich zum Alleröd nach den bisherigen Befunden gekennzeichnet durch zumindest jahreszeitliçh auftretende höhere Abflussmengen und deutlich höhere Abflussniveaus (ca. 1 bis $3 \mathrm{~m}$ ) sowie eine verstärkte fluviale Dynamik.

In einigen Profilen wechselt die Dynamik der Prozesse in der Dryas-III von Erosion zu limnischer und schließlich fluvialer Sedimentation innerhalb der Täler. Im Vergleich zu den rezenten Verhältnissen waren die Fließgewässer während der Dryas-III um ein Vielfaches breiter, die Abflussniveaus lagen zeitweise ca. 1 bis 1,5 $\mathrm{m}$ höher.

Erstmals ist für das Untersuchungsgebiet Vorpommern der Nachweis von Erosionsdiskordanzen in pleni- und spätglazialen fluvialen Sedimenten gelungen. Deren chronologische Einordnung beweist die Existenz von Erosionsphasen während der Dryas-III und des Frühholozäns.

\section{[Lateglacial fluvio-limnic processes in small valleys in Vorpommern (NE Germany)]}

\footnotetext{
Abstract: The data presented in this study result from investigations in a gas-pipeline ditch north of the Pomeranian terminal moraines crossing the till plains of the Mecklenburgian stade.

Small valleys and basin, dominated by peat formation during the Alleröd, were filled with minerogenic sedi-

*)Anschriften der Verfasser: Dr. H. HelBIG, Geologisches Landesamt Sachsen-Anhalt, Köthener Str. 34, 06118 Halle und drs. P. DE KLERK, Geographisches Institut der Ernst-MoritzArndt-Universität, Jahnstr. 16, 17487 Greifswald
}

ments (mainly fine sand, often with silt portions) during Dryas-III. This period, in comparison with the preceding Alleröd, is characterized by, at least seasonally, higher discharges, substantial higher water tables (about 1 to $3 \mathrm{~m}$ ) and stronger fluvial dynamics.

In some investigated profiles, processes within the Dryas-III changed from erosion via limnic to fluvial sedimentation.

In comparison with present conditions, the channels during Dryas-III were substantially wider; water tables were about 1 to $1.5 \mathrm{~m}$ above present levels.

This study presents the first described erosional discordances in pleni- and lateglacial fluvial sediments in Vorpommern: dating of these discordances proves erosional phases during Dryas-III and the Early Holocene.

\section{Problemstellung}

Die Forschungen zu fluvialen Prozessen in Vorpommern waren in der Vergangenheit im Wesentlichen auf große Talungen beschränkt, wobei insbesondere die markanten Abflussbahnen des Haffstausees eine Rolle spielten (JANKE \& REINHARD, 1968; JANKE, 1978). In jüngster Zeit beschäftigten sich auch KAISER \& JANKE (1998), KAISER et al. (1999) sowie Kaiser \& Terberger (1996), BILLWITZ et al. (1998) und BiLLWITZ et al. (2000) mit der spätglazialen fluvialen Morphodynamik in Vorpommern.

In den Jahren 1994 bis 1997 hatten die Autoren Gelegenheit, im Rahmen eines DFG-Schwerpunktprogramms zum, ,Wandel der Geo-Biosphäre seit den letzten 15.000 Jahren - terrestrische Sedimente als Ausdruck sich wandelnder Lebensbedingungen" unter anderem Abschnitte eines etwa $50 \mathrm{~km}$ langen Aufschlusses zu untersuchen (Helbig, 1999 a, b). Dieser Erdgasleitungsgraben führte von Dargun über Grimmen nach Ahrendsee südlich von Stralsund. Im Zuge dieser Bauarbeiten wurden u.a. mehrere kleinere Täler durch einen maximal $200 \mathrm{~cm}$ tiefen Graben aufgeschlossen.

Ziel dieses Aufsatzes ist einerseits die Präsentation und Interpretation von eigenen Befunden aus dem landschaftsgenetisch bisher wenig untersuchten vorpommerschen Binnenland und andererseits der Versuch einer zusammenfassenden Darstellung der spätglazialen fluvial-limnischen Morphodyna- 
mik in Vorpommern auf Grundlage der eigenen und bereits veröffentlichter Ergebnisse sowie deren Diskussion im überregionalen Kontext.

\section{Methoden}

Die Aufnahme und Beschreibung der Bodenprofilaufnahmen und Sondierungen erfolgten nach Bodenkundlicher Kartieranleitung 1994 (AG BoDEN, 1994). Alle Aufschlüsse sind durch Zeich- nungen und Fotos dokumentiert worden.

Die Korngrößenanalysen wurden gemäß der kombinierten Sieb-Pipettmethode nach KÖHN durchgeführt (BARSCH \& BILLwITZ, 1990). Die Bestimmung des Kalkgehaltes und Glühverlustes richtete sich nach BARSCH \& BILlwITZ (1990). Die Vorgehensweise bei der Analyse der Quarzkörner ist in Helbig (1999-a) dargestellt.

Die Proben für die Pollenanalyse wurden aufgear-

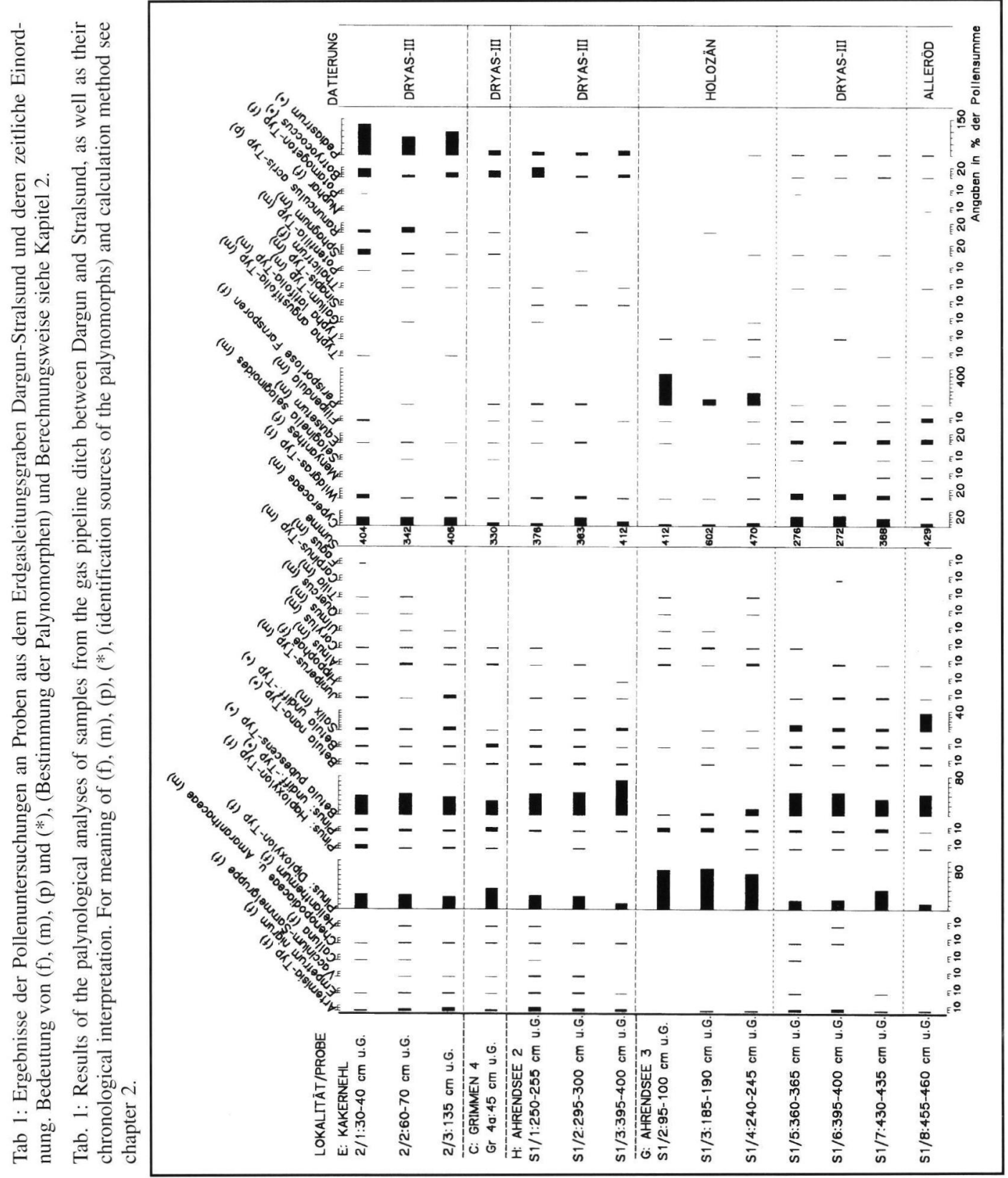


beitet mit $\mathrm{HCl}$, gekocht in $\mathrm{KOH}(20 \%)$, gesiebt $(120 \mathrm{~mm})$, mit HF behandelt und acetolisiert (vgl. FÆGri \& IVERSEN, 1989).

Die Ergebnisse der Pollenanalysen sind in Tabelle 1 dargestellt. Als Berechnungsbasis wurde eine Pollensumme benutzt, die ausschließlich aus Pollentypen von Bäumen/Sträuchern (AP) und terrestrischen (,upland“) Kräutern (NAP) besteht. Die Spalte 'Summe' zeigt die absolute Anzahl der Pollen innerhalb der Summe. Pollentypen, die von Pflanzen nasser bzw. feuchter (,wetland") Standorte produziert werden sowie Typen, die sowohl von „upland“ als ,wetland“ Pflanzen stammen können (z.B. Pollen von Cyperaceae und Poaceae), wurden aus der Summe ausgeschlossen.

Die Pollentypen sind identifiziert und benannt nach FAEGr (1993) (f), Moore et al. (1991) (m) und PunT \& BLACKMORE (1991) (p). Daneben wurden mit $\left(^{*}\right)$ gekennzeichnete Pollentypen unterschieden: Pinus undiff. Typ (nicht eindeutig dem Pinus: Diploxylon-
Typ oder dem Pinus: Haploxylon-Typ zuzuordnen), Betula nana- (kleines Vestibulum), Betula pubescens(großes Vestibulum) und Betula undiff.-Typ (Vestibulum wegen Beschädigungen oder Falten nicht klar sichtbar). Zusätzlich sind noch zwei Algentaxa identifiziert worden: Botryococcus und Pediastrum.

Die Datierung der Proben wurde durch Vergleich mit den vorpommerschen Referenzdiagrammen 'Hoher Birkengraben' (BILwITZ et al., 2000; KAISER et al., 1999; DE KLERK, im Druck) und 'Reinberg C' (DE KLERK et al., im Druck) vorgenommen. $\mathrm{Da}$ in der wissenschaftlichen Literatur die Verwendung der Begriffe für bestimmte Vegetations- und Klimaphasen im Spätglazial nicht einheitlich erfolgt (vgl. BoCK et al., 1985; KAISER et al., 1999; UsINGER, 1985, 1998; LitT \& SteBich, 1999; MerKT \& MüLLER, 1999; SCHIRMER, 1999; HoEk, 1997), ist eine kurze Erläuterung der benutzten Terminologic notwendig. In diesem Text werden die Begriffe „Alleröd“ und „Dryas-III" benutzt, um verschiedene Phasen der Vegetationsentwicklung in Vorpommern anzudeuten (vgl. BILLWITz et al., 2000; KAISER et al., 1999), wobei das Alleröd einer spätglazialen Waldphase und die Dryas-III einer (relativ kalten) offenen Vegetationsphase entspricht. Die Dryas-III schließt das Spätglazial ab. Der Terminus ,spätglaziale Warmphase" ist klimatologisch zu verstehen und bezeichnet sowohl alle vor dem Alleröd liegenden spätglazialen Phasen als auch das bewaldete Alleröd selbst (Lateglacial Interstadial sensu LOWE \& GRAY, 1980; LOWE \& NASP MEMBERS, 1995; WALKER 1995).

\section{Einführung in das Untersuchungsgebiet}

Das Untersuchungsgebiet (Abb. 1) liegt nördlich der Rosenthaler Staffel und wird von den Grundmoränenplatten des Mecklenburger Stadiums (RÜHBERG, 1987) geprägt. Für die Eisfreiwerdung dieses Raumes existieren bisher keine genauen Daten. In

Abb. 1: Untersuchungsgebiet mit Lage der Profile.

Fig. 1: study area and location of described profiles. 
erster Näherung kann der Beginn des Abtauens etwa zwischen 15-14 ka BP angenommen werden (BILLWTTZ et al., 1998).

Die NW-SO verlaufenden Eisrandlagen geben mit ihren Sandern und Schmelzwasserrinnen eine erste Zonierung der Landschaft vor. Nach der von JANKE (1996) in Anlehnung an HurTiG (1957) vorgestellten Gliederung Mecklenburg-Vorpommerns in Großlandschaften ist das hier vorgestellte Unter- suchungsgebiet weitestgehend identisch mit den „ebenen bis flachwelligen, von Tälern durchzogenen Lehmplatten Vorpommerns und des küstennahen Mecklenburgs".

Abbildung 1 macht deutlich, dass im Untersuchungsgebiet ein jungmoränentypisch sehr unübersichtliches Entwässerungsnetz mit vielen Binneneinzugsgebieten ausgebildet ist. Es folgt in seiner Netzstruktur tendenziell dem NW-SO gerichteten Verlauf der Eisränder und den häufig rechtwinklig dazu vorhandenen Spalten und subglazialen Entwässerungsrinnen.

Die hier vorgestellten Aufschlüsse befanden sich ausschließlich innerhalb kleiner Talungen und beckenartiger Senken. Sie gehören rezent zu den Einzugsgebieten der Trebel und des Strelasunds. Zur Frage der Einzugsgebiete ist anzumerken, dass sich im Pleni- und Spätglazial die Entwässerungsrichtungen von den rezenten zumal durch künstliche Verbindungen veränderten Gegebenheiten unterschieden haben können. Ein Beispiel dafür liefern KaISER \& JANKE (1998: 89), die im Rycktal eine der heutigen nordöstlichen Abflussrichtung entgegengesetzte Richtung nach Südwest für die Dryas-III ermittelt haben.

\section{Beschreibung und Interpretation der Gelände- und Labor- befunde}

Medrow 2 (Abb. 2)

Diese Lokalität befindet sich östlich des Dorfes Medrow. Das durch das Profil angeschnittene Fließgewässer verbindet den Ibitzgraben mit der 


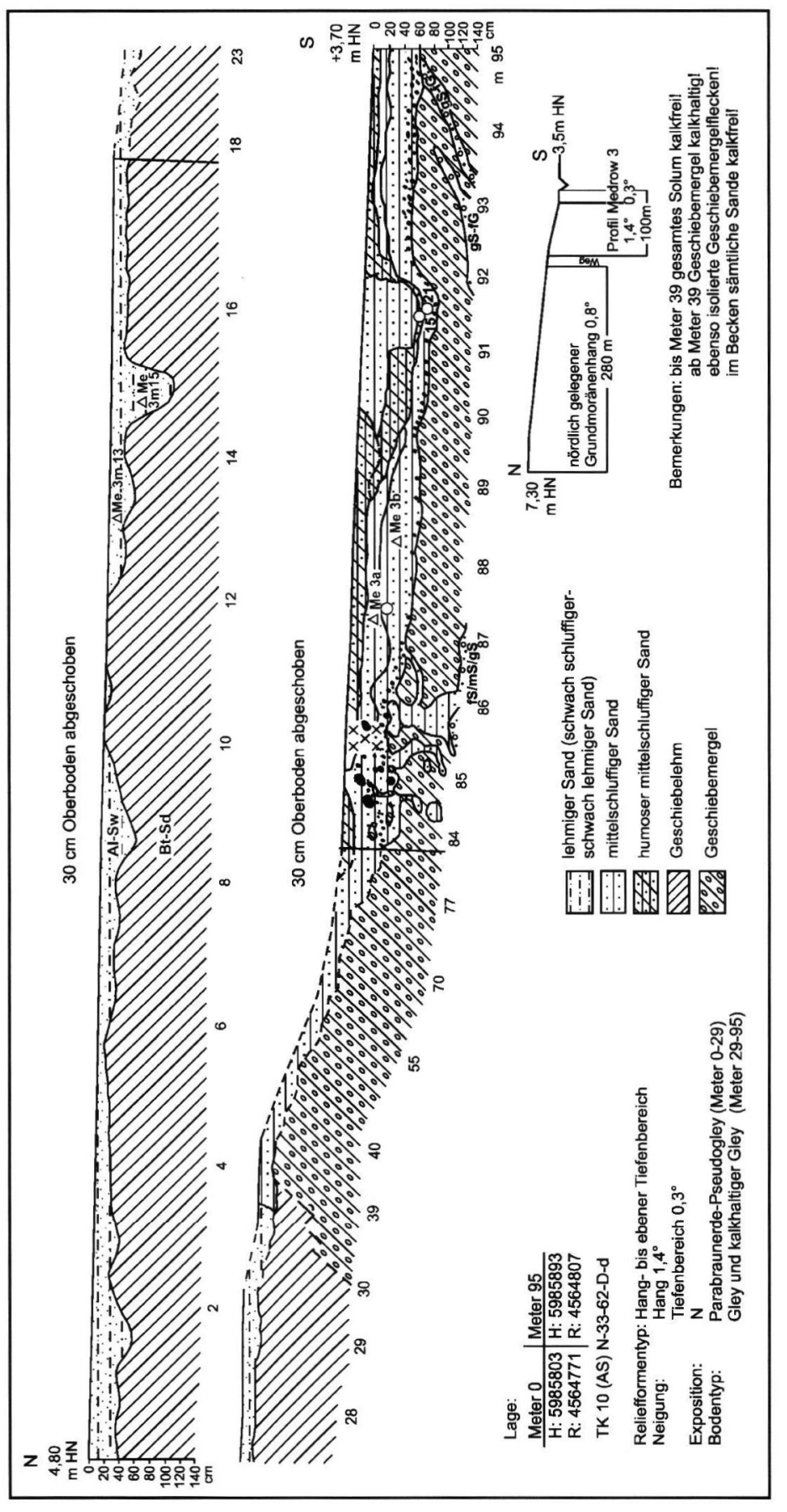

de greifen etwa $25 \mathrm{~m}$ über den rezenten Bachlauf auf die Grundmoräne aus, bis auf eine Höhe von ca. 6,50 $\mathrm{m}$ HN. Das rezente Abflussniveau liegt bei etwa $5,00 \mathrm{~m}$ HN. Demzufolge lag das diesen Sedimenten entsprechende Abflussniveau mindestens $150 \mathrm{~cm}$ über dem heutigen. Spuren einer Erosion der Grundmoräne sind zwischen Meter 0 und 7 in Form von groben Sanden mit Kiesen und Steinen sichtbar. An drei Stellen sind Aufpressungen eines älteren glazifluvialen, schwach lehmigen Sandes in den darüberliegenden Geschiebemergel beobachtet worden. Es ist festzuhalten, dass der schluffige Feinsand im Hangenden fluvial-limnischen Ursprunges ist. Seiner Ablagerung ging eine Erosionsphase voraus. Die Aufpressungen des glazifluvialen Sandes werden mit Bodenfrostprozessen in Verbindung gebracht. Für dieses Profil existieren keine Datierungen. In Analogie zu den folgenden Befunden können wir allerdings mit einiger $\mathrm{Si}-$ cherheit vermuten, dass der hangende schluffige Feinsand während der Dryas-III abgelagert wurde.

\section{Medrow 3 (Abb. 3)}

Medrow 3 liegt nur $600 \mathrm{~m}$ nördlich Medrow 2 und gehört zum gleichen Grabensystem. An dieser Stelle konnte auch der Übergang zur Grundmoräne aufgenommen werden. Das hangende

Trebel. Im Profil ist der südliche Talabschnitt zu sehen. Das rezente Gewässer befindet sich nur wenige Meter nördlich des Meters 0 . Über der Grundmoräne liegen ca. $60 \mathrm{~cm}$ mächtige schluffige, teilweise humose Feinsande (Su3; 64\% Sand, $28 \%$ Schluff, $8 \%$ Ton). Diese schluffigen Feinsan-
Sandpaket ist texturell zweigeteilt: unten ein mittelsandiger Feinsand (96\% Sand, weniger als 1\% Schluff, 4\% Ton), oben ein mittelschluffiger Feinsand (60\% Sand, 33\% Schluff, 7\% Ton). Die liegende Grundmoräne wurde offenbar vorher flächenhaft erodiert. An ihrer Oberkante ist eine 


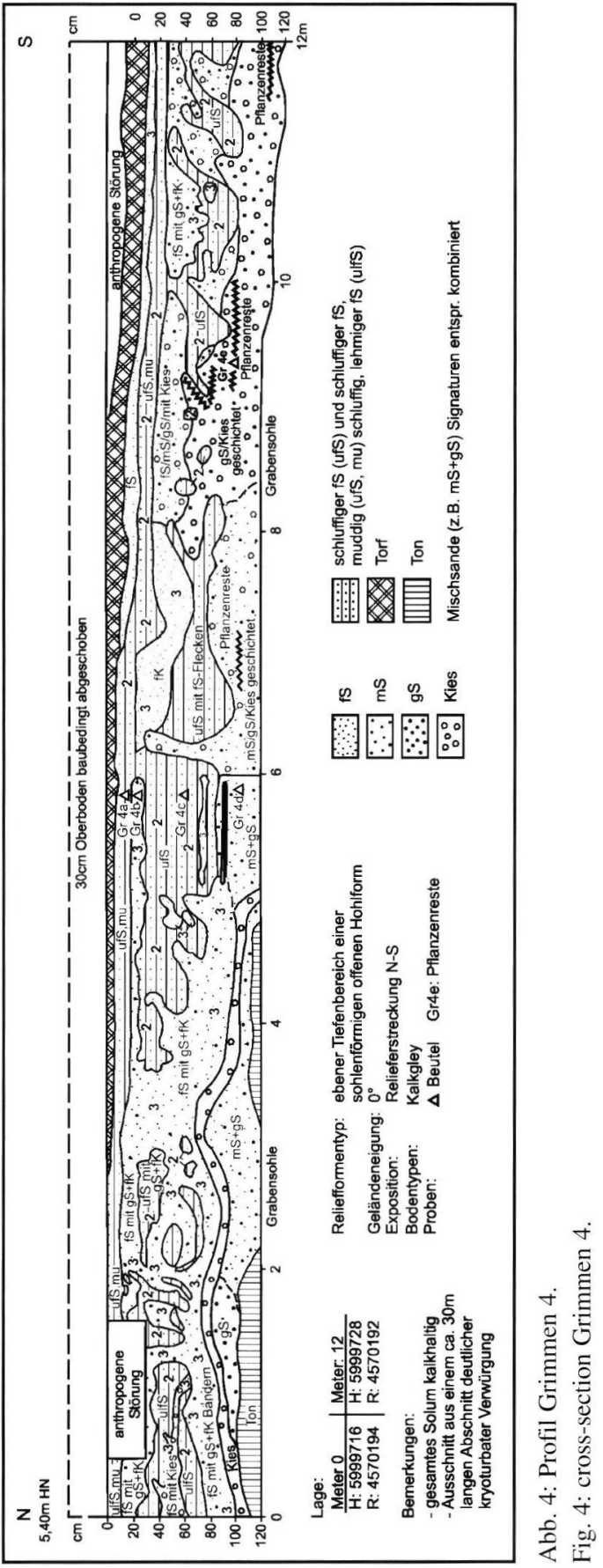

Kies- und Steinsohle ausgebildet. Es zeigen sich bei Meter 85 wieder kryoturbate Verwürgungen des Feinsandes mit dem Geschiebemergel. Der Talsand geht allmählich in den Geschiebedecksand der Grundmoräne über. Oberhalb Meter 29 ist im Geschiebelehm ein Bt-Sd-Horizont ausgebildet, $a b$
Meter 29 talwärts ein Go/Gr-Horizont. Der Geschiebedecksand ändert bei diesem Bodentypenwechsel seinen Charakter zunächst nicht. Zwischen Meter 36 und 39 steigt die Entkalkungsgrenze nach oben (Wechsel von Geschiebelehm zu Geschiebemergel) und gleichzeitig wird der Geschiebedecksand vom Talsand abgelöst. Geschiebedecksand und Talsand sind durch ihre spezifischen Texturen eindeutig voneinander zu unterscheiden: schlecht sortierte lehmig-schluffige Sande der Grundmoräne im Vergleich zu sehr feinkörnigen schluffigen Sanden des fluvial-limnischen Milieus. Allerdings lassen die Korngrößenanalysen des Geschiebedecksandes (Meter 13/15) eine Beeinflussung durch den Talsand vermuten. Insbesondere die Probe aus der sackartigen Vertiefung des Geschiebedecksandes (Me3m15) legt nahe, dass die Sedimentation der Talsande zeitweise bis in diesen Bereich erfolgte. Die Talsande greifen auf dieser Talseite ca. $60 \mathrm{~m}$ über den rezenten Bach auf die Grundmoräne bis auf eine Höhe von ca. 4,50 $\mathrm{m} \mathrm{HN}$ aus. Das rezente Abflussniveau liegt bei etwa $3,00 \mathrm{~m} \mathrm{HN}$.

Der Profilaufbau ist dem der Lokalität Medrow 2 sehr ähnlich. Auch hier ist eine Sedimentation der feinen Sande während der Dryas-III zu vermuten. Da eine Probe für die Pollenanalyse aus dem hangenden humosen mittelschluffigen Sand keine Pollen enthielt, war eine palynologische Datierung nicht möglich.

Grimmen 4 (Abb. 4)

Grimmen 4 liegt wenige Kilometer östlich von Grimmen und gehört zur Niederung des Schwedengrabens, der in die Trebel entwässert. In den relativ groben Sanden und Kiesen im unteren Bereich des Profils fanden sich zahlreiche fossile Holz- und Pflanzenreste. Über dem groben $\mathrm{Ma}$ terial folgt ein schluffiger Feinsand. Wegen der Kryoturbationen ist die Schichtenfolge nicht leicht zu erkennen. Sehr wahrscheinlich lagert über dem schluffigen Feinsand wieder ein etwas gröberes Material (Feinsand), gefolgt von muddig-schluffigem Feinsand und schließlich von Torf. Für die zeitliche Einordnung der Prozesse konnte eine Pollenprobe aus dem muddeartigen schluffigen Feinsand ( $\mathrm{Gr} 4 \mathrm{a})$ ausgewertet werden. Diese zeigt ein Pollenspektrum aus der Dryas-III.

Es ist sehr wahrscheinlich, dass die Holzreste im unteren Profilteil aus dem Alleröd stammen und während einer jungdryaszeitlichen fluvialen Phase transportiert und sedimentiert wurden. Offenbar nehmen die Dryas-III-Sande einen erheblichen Teil der Talbreite ein, so dass wiederum ein ge- 


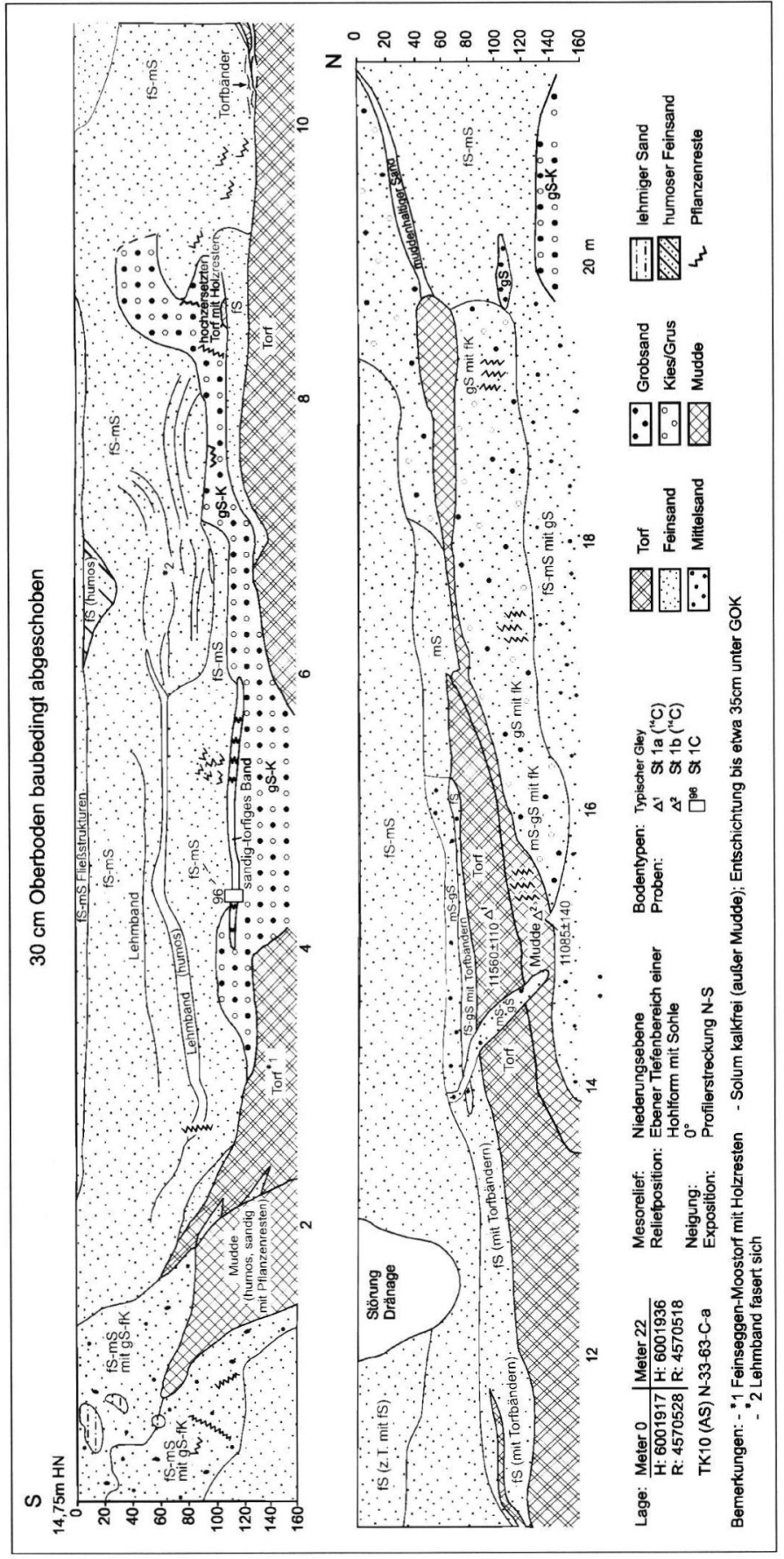

Als Fazit bleibt festzuhalten, dass in diesem Bereich wieder minerogene fluviale und fluviallimnische Sedimente der DryasIII vorgefunden wurden, die eine wechselnde Transportdynamik bezeugen (Kies- bis schluffige Feinsandschichten) und vermutlich postsedimentär unter Mitwirkung von zumindest saisonaler Bodengefrornis während der Dryas-III deformiert wurden.

Stoltenhagen 1 (Abb. 5)

Das Profil Stoltenhagen 1 wurde wenige Meter entfernt von einem Graben aufgenommen, der in die Kronhorster Trebel entwässert. Rezent steht dieser Bereich mit dem Fließgewässer nicht in Verbindung. In unmittelbarer Nähe befindet sich eine kleine vermoorte Senke. Bei dem Profil handelt es sich um ein ehemaliges Becken mit einem Durchmesser von ca. $20 \mathrm{~m}$. Es ist eingebettet in fluviale Sande. In unmittelbarer Nachbarschaft zu diesem Profil wurden zwei ähnliche Senken beobachtet. Zwei ${ }^{14} \mathrm{C}$-Proben aus der liegenden Mudde $\left({ }^{14} \mathrm{C}\right.$-Alter*) $11085 \pm 140$; cal BC 1119510905; Labornummer $\mathrm{Hv}$ 20631) und dem hangenden Feinseggen-Moostorf $\left({ }^{14} \mathrm{C}-\mathrm{Al}-\right.$ ter*) $11560 \pm 110$; cal BC 11690 11395; Labornummer $\mathrm{Hv}$ 20632) sind als Mischproben entnommen worden. (*)Die bei Helbig (1999a) auf Seite 85 eingetragenen Daten sind falsch. Sie beruhen auf einer mündlichen Mitteilung aus dem Labor. Die so mitgeteilten Daten wurden später vom Labor durch den schriftlichen Bericht korrigiert.) Die Ursache für das jüngere

genüber dem heutigen Abflussniveau höherer Wasserstand während der Dryas-III zu vermerken ist.

Die eindrucksvollen Kryoturbationen treten vor allem als diapirartige Aufpressungen der groben Sande in die schluffigen Sande in Erscheinung.
Datum der Mudde ist wahrscheinlich im Vorhandensein jüngeren Wurzelmaterials zu suchen. Das Becken verlandete im Verlaufe des Alleröd. Offenbar wurde der Torf in der anschließenden fluvialen Phase während der Dryas-III zumindest zwischen Meter 4 und 6 erodiert. Über den organo- 


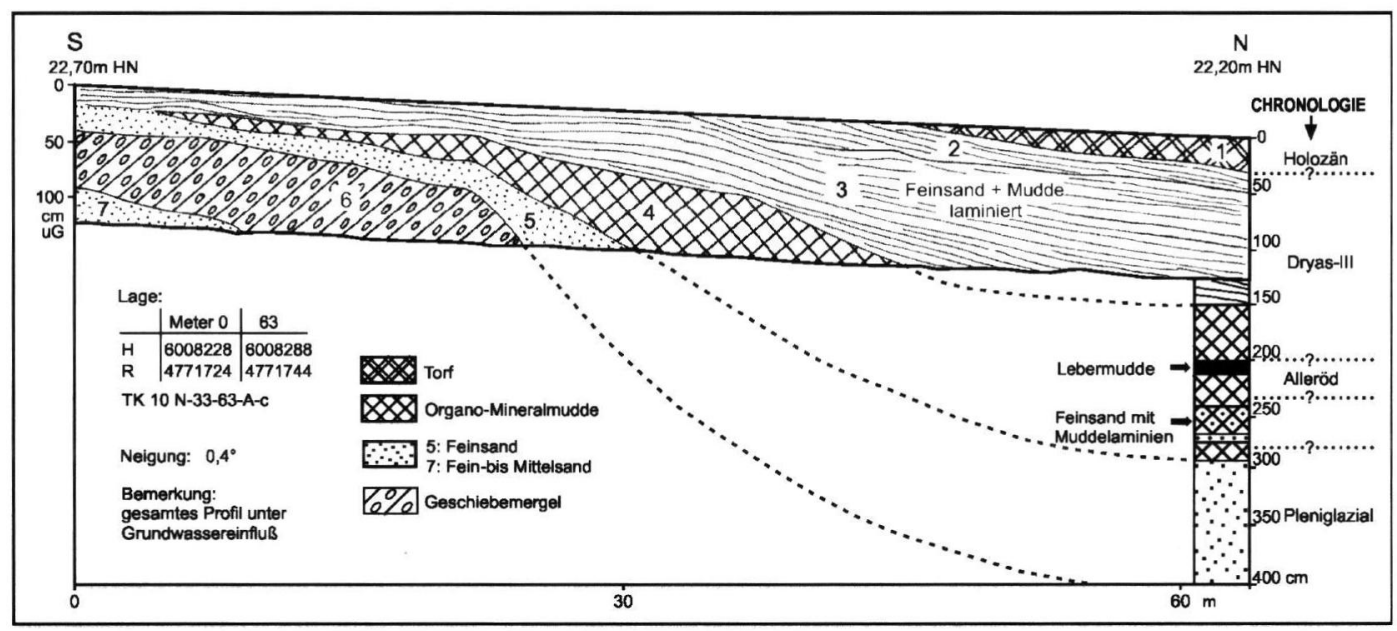

Abb. 6: Profil Kakernehl 2.

Fig. 6: cross-section Kakernehl 2.

genen Lagen sedimentierten zunächst relativ grobe Sande und anschließend ein Fein- bis Mittelsand. Vor allem in den gröberen Sanden sind Reste aufgearbeiteten Torfmaterials eingelagert. In der Fein- bis Mittelsandschicht wurden Lehmbänder gefunden.

Insgesamt lässt sich feststellen, dass eine im Alleröd vorhandene Sumpflandschaft während der Dryas-III durch fluviale Sande überdeckt wurde. Allerdings ist zwischen der interstadialen organogenen Sedimentation und Sedentation und der stadialen minerogenen Sedimentation eine Erosionsphase eingeschaltet. Dieser Aufschluss liefert erneut einen Hinweis auf höhere Wasserstände während der Dryas-III. Der Höhenunterschied zwischen der Oberkante des Allerödtorfes im Randbereich des Beckens und der Oberkante der DryasIII-Sande beträgt ca. $90 \mathrm{~cm}$ (ca. $30 \mathrm{~cm}$ Oberboden wurden baubedingt abgeschoben).

Kakernehl 2 (Abb. 6)

Kakernehl 2 gehört zum Einzugsgebiet des Greifswalder Boddens. Das Profil liegt am Rand einer vermoorten Niederung. Wenige Meter nördlich des aufgenommenen Profils befindet sich ein kleines Fließgewässer. Die Besonderheit der spätglazialen Sedimentation besteht hier in dem Vorhandensein laminierter Sedimente (Feinsand- und Muddelaminen in Wechsellagerung). Diese FeinsandMudde-Schichtung ist bei Meter $60 \mathrm{ca} .120 \mathrm{~cm}$ mächtig, darunter folgen etwa $50 \mathrm{~cm}$ Mineral-Organomudde, die teilweise ebenfalls Lamination aufweist. Jede der Laminen ist weniger als $1 \mathrm{~cm}$ stark. Der Feinsand ist nach Analyse einer Probe unter dem Rasterelektronenmikroskop nicht äo- lisch transportiert. Die Laminierung lässt ohnehin eine jahreszeitlich gesteuerte Ablagerung in einem an das Fließgewässer angeschlossenen Stillwasserbecken vermuten, wobei die Muddeschichten sich während ruhiger Stillwasserphasen bilden konnten, die Feinsandlaminen dagegen den fluvialen Eintrag repräsentieren. Die Morphologie des nur unmerklich in die umliegenden ebenen Grundmoränenplatten eingesenkten Beckens sowie die relativ gute Sortierung der minerogenen Lagen sprechen gegen einen kurzstreckigen Transport und Eintrag des minerogenen Materials von den umliegenden Platten.

Die Pollenbefunde (Tab. 1: Kakernehl 2/1, 2/2 und $2 / 3$ ) zeigen, dass die laminierten Sedimenten in die Dryas-III zu stellen sind. Die zusätzliche Anwesenheit von Pollen wärmeliebender Taxa (z.B. Corylus, Ulmus, Quercus, Tilia und Fagus) ist ein Resultat von Fernflug und/oder Umlagerung älteren Materials; dies gilt auch für die hohen Pollenwerte von Pinus: Haploxylon-Typ, besonderes in Probe Kakernehl 2/1. Im Becken waren ebenfalls Botryococcus und Pediastrum vertreten, während die Anwesenheit von Wasserhahnenfuß aus den Werten für den Ranunculus acris-Typ geschlussfolgert werden kann.

Unter den laminierten Schichten, die einen erheblichen minerogenen Anteil enthalten, folgen Leber- und Mineral-Organomudden, die in Analogie zu den typischen lithostratigraphischen Verhältnissen in Vorpommern wohl dem Alleröd zuzurechnen sind. Im Liegenden befinden sich neben fossilfreien Sanden weitere geringmächtige organogene Schichten. Eine Pollenprobe aus diesem Bereich (Kakernehl 2/4) brachte keine aus- 
wertbaren Ergebnisse.

Auch an dieser Lokalität wird eine sehr wahrscheinlich allerödzeitliche Seenphase mit organogenen Mudden von einer energiestärkeren fluvial-limnischen jungdryaszeitlichen Fazies abgelöst. Die Besonderheit besteht hier in einer zeitweise während der Dryas-III bestehenden spezifischen Beckensituation, die zu einer jahreszeitlich induzierten Schichtungsrhythmik führte. Ein Vergleich der Höhenlage des Allerödtorfes (durch Auflast komprimiert!) und Dryas-III-Schichten offenbart einen Höhenunterschied von ca. $150 \mathrm{~cm}$.

\section{Ahrendsee 1 (Abb. 7).}

Alle Ahrendseeprofile gehören zum Einzugsgebiet des Strelasunds und liegen jeweils nur ca. 1 $\mathrm{km}$ voneinander entfernt. Bei Ahrendsee 1 konnte der Übergang eines Tales zur Grundmoräne aufgenommen werden. Der rezente Graben befindet sich unmittelbar südlich des Profils. Ähnlich wie bei Medrow 3 vollzieht sich der Übergang vom Geschiebedecksand zum Talsand allmählich. Beide Sande sind nach Schichtung und Textur deutlich unterscheidbar. Die fluvial-limnischen Talsande sind im Liegenden des Aufschlusses als tonige Feinsande und darüber als Fein- bis Grobsande ausgebildet. Neben Parallelschichtung sind im oberen, gröberen Material stellenweise Schräg- schichtungen vorhanden. Etwa bei Meter 200 wurden wieder Holzrestlagen in den dort parallel geschichteten Sanden gefunden. Im Gegensatz zu vertikal eingewachsenen jungen Wurzelresten wurden diese Holzrestlagen zweifelsfrei fluvial transportiert und anschließend sedimentiert. Wir können diese Sande deshalb mit großer Wahrscheinlichkeit in den letzten Abschnitt des Spätglazials, die Dryas-III, stellen (s. a. Grimmen 4). Wie in der Profilzeichnung zu sehen ist, nehmen auch hier die spätglazialen Talsande einen erheblich größeren Raum ein, als der rezente Graben vermuten lässt. Die spätglazialen fluvial-limnischen Sande reichen bis in eine Höhe von ca. 19,50 m HN. Das rezente Abflussniveau des nächst liegenden Grabens liegt bei $18,50 \mathrm{~m} \mathrm{HN}$.

\section{Ahrendsee 3 (Abb. 8)}

Das Profil Ahrendsee 3 befindet sich am Rand einer Grundmoräne am Übergang zu einer Sandplatte unweit Ahrendsee 2. Das morphologisch deutlich ausgeprägte Tal ist durch eine kolluviale Ackerrandstufe an den Rändern zusätzlich versteilt und zeigt unter den holozänen Torfen bis auf eine geringmächtige Torflage in Bohrung S1 lediglich limnische (Mudden) und fluviale (Sande) Sedimente. Zunächst wurden die von weitgehend humusfreien Sanden über- und unterlagerten organoge-

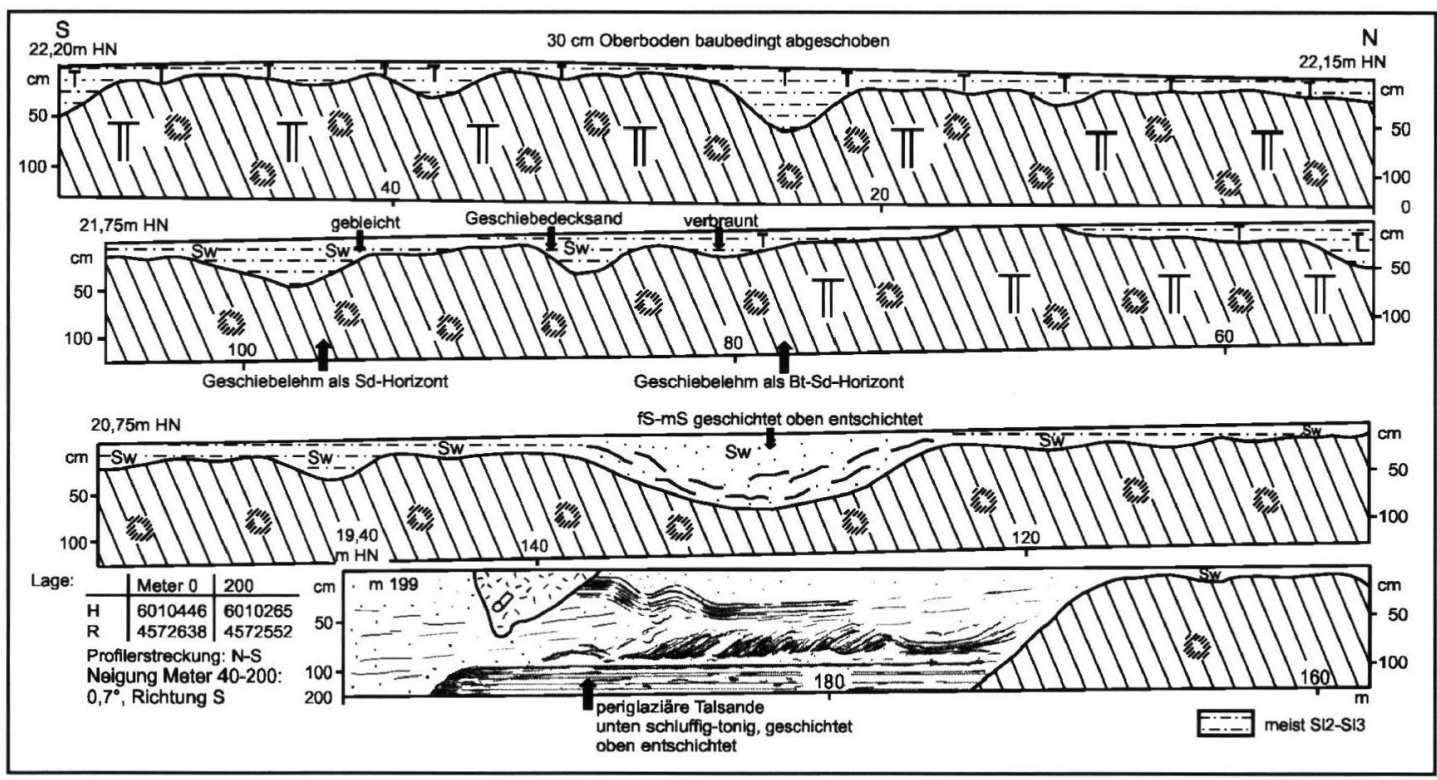

Abb. 7: Profil Ahrendsee 1.

Fig. 7: cross-section Ahrendsee 1. 


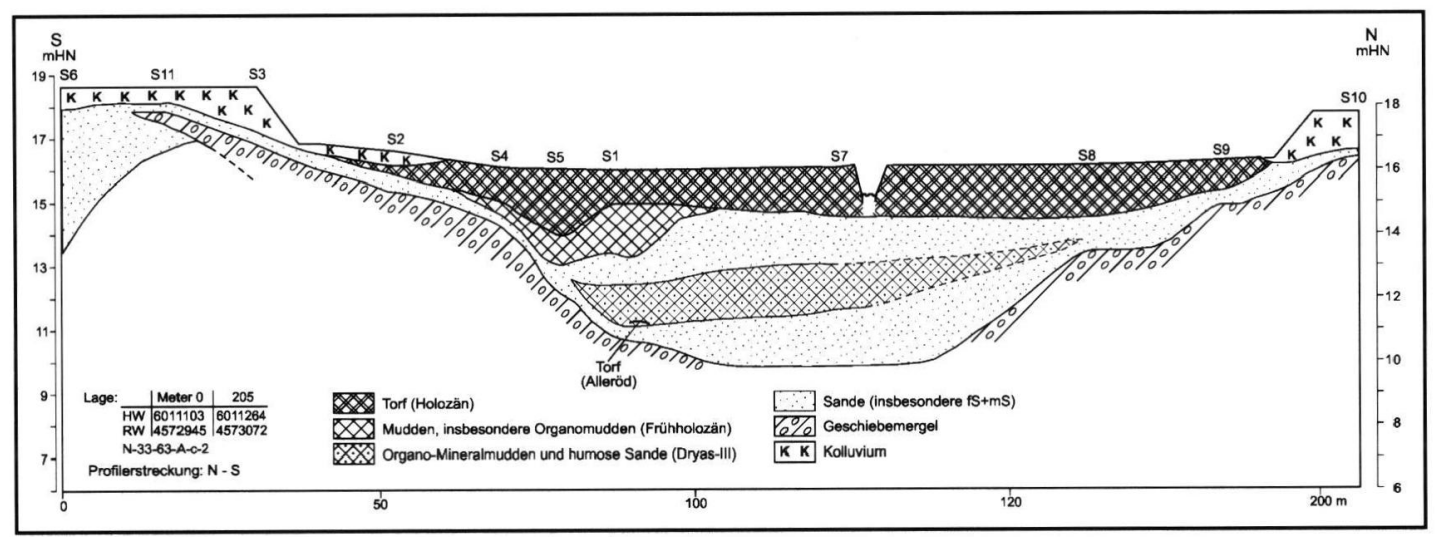

Abb 8: Profil Ahrendsee 3.

Fig. 8: cross-section Ahrendsee 3.

nen Mudden und humosen Sande im Zentrum des Tales als interstadiale Bildungen gedeutet (HELBIG, 1999 a). Nach den nunmehr vorliegenden Pollenuntersuchungen muss diese Ansicht korrigiert werden.

Aus Bohrung S1 wurden 7 Pollenproben ausgewertet. Die Probe S1/ 8 wurde aus einer Tiefe von $455-460 \mathrm{~cm}$ u.G. entnommen und entstammt der einzigen im Talquerprofil angetroffenen nichtholozänen Torfschicht (Glühverlust $37 \%, \mathrm{CaCO}_{3}-$ Gehalt $27 \%$ ). Sie ist insgesamt nur $7 \mathrm{~cm}$ mächtig, wird von teilweise humosen Sanden über- und unterlagert und zeigt ein eindeutig allerödzeitliches Pollenspektrum. Die relativ hohen Werte der Pollen von Salix deuten auf die Anwesenheit von Weiden in der unmittelbaren Umgebung der beprobten Stelle (lokale oder extralokale Anwesenheit sensu JANSSEN, 1966), d.h. gegen den Talrand hin. Die „wetland“-Pollentypen suggerieren eine Sumpfvegetation bestehend aus Equisetum und Filipendula. Das Vorhandensein offener Wasserstellen wird angedeutet durch Pollen von Nuphar sowie Botryococcus und Pediastrum.

Die Proben S1/7, S1/6 und S1/5 stammen aus einer laminierten Organo-Mineralmuddeschicht (Glühverlust 3-10 \%, $\mathrm{CaCO}_{3}$-Gehalt 5-12\%) zwischen $360-435 \mathrm{~cm}$ u.G. Sie zeigen ein Dryas-IIIzeitliches Pollenspektrum. Zwischen dem Allerödtorf und den Dryas-III-zeitlichen Mudden liegen in Bohrung S1 noch $44 \mathrm{~cm}$ Fein- bis Grobsande. Es bleibt demzufolge ein Ansteigen des Wasserspiegels und eine Zunahme der geomorphologischen Aktivität nach dem Alleröd festzuhalten. Wahrscheinlich ist ein Teil der allerödzeitlichen Torfe während der Dryas-III erodiert worden.
$\mathrm{Zu}$ dieser Aktivitätsphase sind auch die hangenden Sande (bei S1 zwischen 290 und $360 \mathrm{~cm}$ u.G.) zu zählen. Sie wurden am Ende der Dryas-III abgelagert.

In einer rinnenähnlichen Struktur (im Bereich S1 und S5) wurden Mudden (100-235 cm u.G.: teilweise grobdetritische Organomudde, Glühverlust $90 \%$; 235-260 cm u.G.: Organo-Mineralmudde, Glühverlust $30 \%, \mathrm{CaCO}_{3}$-Gehalt $7 \%$ ) abgelagert (Pollenproben S1/4 und S1/3), welche von einer holzreichen Torfschicht (Probe S1/2) abgedeckt werden. Diese drei Pollenproben zeigen ein frühholozänes Pollenspektrum mit einer extralokalen Überrepräsentation des Pinus: Diploxylon-Typ. Solche extralokalen Niederschläge von Pinus-Pollen werden sehr oft in den frühholozänen Ablagerungen der vorpommerschen Täler registriert, z.B. im Peenetal (HALLIK, 1944; KLOSS, unveröff. Material in FISCHER, 1995; JANKE, unveröff. Material in HelKe, 1999), im Recknitztal (Kraus, 1999; MICHAELIS, 2000; ReINHARD, 1963) und im Rycktal (WeRTH \& BAAs, 1936). Für das frühholozäne Vorpommern ist somit die Bedeutung der Kiefer als wichtiger Bestandteil der Vegetation an den trockeneren Standorten im Talgrund bzw. an den Talrändern abzuleiten. Die ,wetland“-Typen suggerieren eine Sumpfvegetation bestehend aus Farnen mit Equisetum, Filipendula und Typha. Wegen der extralokalen Überrepräsentation von Pinus ist es sehr schwierig diese Pollenproben zeitlich genau einzuordnen. Auf Grund der Anwesenheit solcher Pollentypen, die sonst typischerweise nicht zu Beginn des Holozäns auftreten (z.B. Alnus, Corylus, Ulmus, Quercus und Tilia), ist anzunehmen, dass die Sedimente des aller ersten Holozänabschnittes fehlen. Damit dürfte die rinnenartige 
Struktur im Bereich S1 und S5 das frühholozäne Flussbett darstellen, durch Tiefenerosion in die Sande der Dryas-III eingeschnitten.

Nach der limnischen Verfüllung des ehemaligen Flussbettes entwickelte sich im Tal ein Flusstalmoor, in dem im Vergleich zu den Verhältnissen während der späten Dryas-III nur wenig Wasser transportiert wurde.

Im Vergleich zu den rezenten geringen Abflussmengen, die lediglich in der Lage sind einen kleinen Graben zu speisen, ist während des Spätglazials und besonders während der Dryas-III zumindest jahreszeitlich die gesamte Talbreite genutzt worden. Die genauen maximalen Sedimentationshöhen der Dryas-III-Sande und des Allerödtorfes lassen sich in diesem Profil nicht rekonstruieren, da beide erodiert sein können. Als Hinweis in diesem Zusammenhang kann die Höhendifferenz zwischen Allerödtorf und Oberkante Dryas-III-Sande gelten. Sie beträgt etwa $200 \mathrm{~cm}$.

Ahrendsee 2 (Abb. 9)

Durch das Profil Ahrendsee 2 wurde an der Südseite der $20 \mathrm{~m}$ lange Übergangsbereich von einer Sandplatte in einen Talbereich erfasst. Die Pollenproben aus der Bohrung S1 (S1/1 bis S1/3) zeigen, dass sowohl die Kalkmudde (Glühverlust $17 \%, \mathrm{CaCO}_{3}$-Gehalt $63 \%$ ) als auch die darüber liegenden Organo-Mineralmudden (Glühverlust 4-13\%, $\mathrm{CaCO}_{3}$-Gehalt 7-16\%) während der Dryas-III abgelagert wurden. Die Sande oberhalb dieser Mudden können deshalb frühestens in ei- ner späten Phase der Dryas-III sedimentiert sein. Überraschenderweise finden sich in diesem Profil keine allerödzeitlichen Ablagerungen. Es sind zwei Überlegungen für das Fehlen allerödzeitlicher Sedimente in Betracht ziehen: entweder war das Tal im Alleröd zu trocken für die Bildung organischer Ablagerungen und/oder sie wurden durch Erosionsprozesse ausgeräumt.

Die Mudden gehen nach oben und zum Talrand in parallel geschichtete Mudde-Feinsand-Sedimente über, die von reinem Sand überlagert werden. Am südlichen Talrand sind die Muddelaminen schräggeschichtet. Sie laufen dabei parallel zur Schichtung im Sand. Dieses Schichtungsbild wird als Sedimentation an einem Gleithang gedeutet, bei der die jahreszeitlich bedingte Muddesedimentation die fluvial vorgegebene Schrägschichtung nachzeichnet. An der Nordseite ist ebenfalls ein Uferbereich angeschnitten worden. Hier geht das Muddepaket (Organo- und Organo-Mineralmudden) aus der Dryas-III zum Talrand hin ebenfalls in eine Mudde-Sand-Wechselschichtung über. Die Schichten laufen parallel. Die über den Mudden sedimentierten Dryas-III-Sande sind vor allem als Fein- bis Mittelsande anzusprechen. Zwischen den organischen Mudden und den liegenden Sanden ist Kalkmudde gefunden worden.

Somit liegen hier vergleichbare Verhältnisse wie an der Lokalität Ahrendsee 3 vor: Einer ersten Phase der Dryas-III mit möglicher Erosion folgte ein Abschnitt mit limnischen Sedimentationsbedingungen (Kalk-, Organo- und Organo-Mineral-

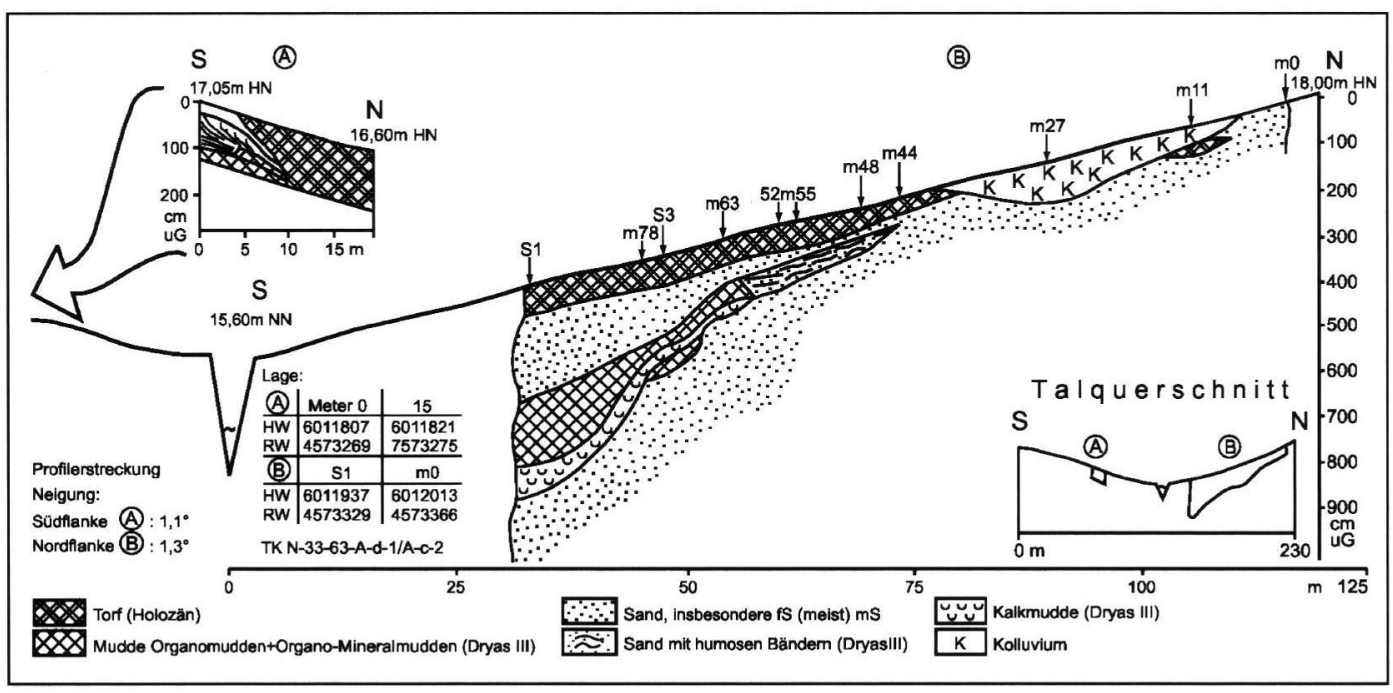

Abb. 9: Profil Ahrendsee 2.

Fig. 9: cross-section Ahrendsee 2. 
mudden) und schließlich eine spätere Phase fluvialer Dynamik mit Sedimentation weitgehend humusfreier Sande wechselnder Körnung.

Die spätglaziale Talverfüllung hat eine Gesamtbreite von etwa $180 \mathrm{~m}$ und reicht bis in eine Höhe von mindestens 17,00 m HN. Das rezente Fließgewässer ist ca. $1,50 \mathrm{~m}$ breit und liegt mit seiner Uferkante bei 15,60 m HN.

\section{Zusammenfassung der Befunde}

Die Täler der Grundmoränenplatten Vorpommerns zeigen für das Spätglazial eine deutliche fluviale und limnische Dynamik. Die vorliegenden Befunde lassen vor allem Schlüsse für den Zeitraum des Alleröd und der darauffolgenden DryasIII zu. Die Dryas-III ist im Untersuchungsgebiet nach unseren Befunden im Vergleich zum Alleröd gekennzeichnet durch deutlich höhere Wasserstände, zumindest jahreszeitlich auftretende höhere Abflussmengen und eine verstärkte fluviale Dynamik. Sedimente der Dryas-III liegen 90 bis $200 \mathrm{~cm}$ über denen des Alleröd (Stoltenhagen 1, Kakernehl 2, Ahrendsee 3) und 100 bis $150 \mathrm{~cm}$ über den rezenten Entwässerungsniveaus (Medrow $2 \& 3$, Ahrendsee $1 \& 2$ ). In den während des Alleröds versumpften und durch Torfbildung geprägten Talabschnitten und Becken erfolgte während der Dryas-III eine mehr oder weniger minerogen geprägte Sedimentation. Im Raum Ahrendsee begann die Dryas-III möglicherweise mit einer Erosionsphase und teilweise fluvialen Sedimenten, gefolgt von einer ausgeprägten limnischen Phase, der sich ein eher fluvial bestimmter Abschnitt anschloss. Im Vergleich zu heute waren während der Dryas-III die Fließgewässer um ein Vielfaches breiter. Nachweisbar ist häufig ein jungdryaszeitliches Sedimentpaket aus Sand, zumeist Feinsand, nicht selten mit deutlichem Schluffanteil. Bei guten Aufschlussverhältnissen zeigen sich in der Regel auch Erosionsdiskordanzen (Erosionslagen aus kiesigem und steinigem Material (Medrow 2 und 3, Grimmen 4) oder erodierte Torfe (Stoltenhagen 1). Zeitlich fixierbar sind bisher nur die Erosion von allerödzeitlichen Torfen im Profil Stoltenhagen 1 während der DryasIII und die frühholozäne Erosion von Dryas-IIIzeitlichen Sanden in Profil Ahrendsee 3. Die pleniglazial angelegten tiefen Schmelzwasserrinnen wurden durch spätglaziale Sedimente erheblich aufgefüllt.

\section{Zusammenfassende Diskussion der spätglazialen fluvial-limnischen Morphody- namik in Vorpommern}

JANKE \& ReINHARD (1968) und JANKE (1978) haben auf der Grundlage ihrer Untersuchungen der Entwicklungsphasen des Talnetzes in Nordostdeutschland erkannt, dass die Täler während des Pleniglazials zunächst als Abflussbahnen der Schmelzwässer (Resteis, Toteis, Haffstausee) dienten und während dieser Zeit im wesentlichen ihre heutige Breite durch Seitenerosion erreichten. Es ist eine starke Sedimentation klastischen Materials festzustellen (Phase 1).

Zwischen Pleniglazial und Beginn des Alleröds wird eine Tiefenerosionsphase der fließenden Gewässer vermutet, wobei die Fließrichtung schon der heutigen entsprochen haben soll. Die Flüsse besaßen ein kräftiges Gefälle und schnitten sich auf einem Teil der vorher gebildeten Talbreite kerbtalartig ein. Die Erosion erfolgte bis in die Nebentäler hinein (Phase 2). JANKE \& Reinhard (1968) und JaNKE (1978) vermuten, dass der Wechsel von Phase $1 \mathrm{zu}$ Phase 2 mit einem sehr tiefliegenden Wasserspiegel im Ostseebecken (Erosionsbasis) zusammenhängt.

Auch in Polen, Litauen und Estland wurden die fluvialen Prozesse während des Pleni- und Spätglazials entscheidend durch die Höhen der Wasserspiegel von Eisstauseen in- und außerhalb des Ostseebeckens gesteuert. Diese erosionsbasisbestimmte Komponente der fluvialen Dynamik des Pleni- und frühen Spätglazials war in den genannten Ländern nicht gleichzeitig wirksam, sondern trat, in Abhängigkeit von ihrer paläogeographischen Lage (Entfernung vom Eisrand bzw. Zeitpunkt der Eisfreiwerdung), zeitlich versetzt in Erscheinung.

So verweist STARKEL (1995) für die Weichsel im Unterschied zu anderen europäischen Flüssen auf eine pleniglaziale Tiefenerosionsphase im Zusammenhang mit dem Absinken der Erosionsbasis durch den allmählichen Rückzug des Eises und zunehmender Kontinentalität. Dvareckas (1995) sieht die Anlage der älteren Flussterrassen in Litauen im Zusammenhang mit Kaskaden von Eisstauseen während der verschiedenen Phasen des Eisrückganges. RAUKAS \& MiIDEL (1995) betonen die Bedeutung der durch den Eisabbau und die dem Eisrand vorgelagerten Eisstauseen gesteuerte Höhe der Erosionsbasis in der Frühphase der Talentwicklung - in Estland bedingt durch den späten Eisabbau im Zeitraum zwischen 13-11 ka BP, also während des Spätglazials. Bis zum Beginn 
des Alleröd hatten sich die Flüsse im estländischen Ostseeraum um 20-80 m eingeschnitten, wobei auch die isostatische Landhebung zu berücksichtigen ist.

In anderen Gebieten Europas führt offenbar der Rückgang der Denudation außerhalb der Flusstäler infolge zunehmender Pflanzenbesiedelung und die damit verbundene Verringerung der Sedimentfracht zwischen 13-11 ka BP zu einer Erosion in den Tälern (VAndenBerghe, 1995).

Das Alleröd und die Dryas-III in Vorpommern sind nach JANKE (1978) gekennzeichnet durch ein schnelles Ansteigen des Grundwasser- und Flussspiegels und durch die Akkumulation von Sanden, Mudden und Torfen (Phase 3). Janke \& ReInHARD (1968) führten diese Entwicklung auf den steigenden Wasserspiegel im südlichen Ostseebecken zurück. Während des späten Alleröd und der DryasIII soll es dabei zu Transgressionen des Baltischen Eisstausees gekommen sein, die teilweise mehrere Meter über HN betrugen. Die Talmündungen wurden dabei fördenartig überflutet. Das Grenzund Peenetal sowie das Ryck- und Ziesetal waren während mindestens eines Teils dieser Phase 3 durch die Transgression bedingt zu einem mit dem Baltischen Eisstausee in unmittelbarer Verbindung stehenden zusammenhängenden See aufgestaut. Die Arbeiten von KaISER \& JANKE (1998) im Rycktal erbrachten Belege für eine stärkere fluviale Dynamik während der Dryas-III, die lithologisch im Gegensatz zum Alleröd als humusfreies minerogenes Sediment erscheint. Für das Alleröd wurde bei Bartmannshagen, Lkr. Nordvorpommern, ein Wasserstand von 1-2 $\mathrm{m}$ HN rekonstruiert. Der Fluss schüttete seine Sande in der Dryas-III mindestens 2-3 m über das vermutete Seeniveau des Alleröd bis in eine Höhe von wenigstens $4 \mathrm{~m} \mathrm{HN}$ und damit ca. 1,5 m über das rezente Entwässerungsniveau. Auch im Endinger Bruch westlich von Stralsund ist eine fluviale, fossilfreie, sandige Fazies der Dryas-III über organogenen limnischen Sedimenten des Alleröd festgestellt worden (KAISER et al., 1999).

Für den vorpommerschen Raum haben bisher alle Autoren übereinstimmend die Akkumulation von zunächst überwiegend organogenem Material während des Alleröds und einen Wechsel zu primär minerogenen Sedimenten während der Dryas-III feststellen können. Anscheinend ist also die Sedimentakkumulation während dieser Phasen verbreitet. Dass allerdings auch mit Tiefenerosionsereignissen zu rechnen ist, zeigen zumindest die Lokalität Stoltenhagen1 und wahrscheinlich auch
Ahrendsee 3, bei denen Allerödtorfe teilweise erodiert worden sind. Eine zweite deutliche Übereinstimmung besteht bezüglich der hohen Abflussniveaus, die nach den bisherigen Befunden während der Dryas-III um etwa 1-3 m über denen des Alleröd und 1-1,5 m über den heutigen gelegen haben. Bemerkenswerterweise ist für das Alleröd eine nicht zu übersehende Versumpfungstendenz vieler Talabschnitte zu verzeichnen. Die DryasIII zeigt in einigen Talabschnitten auch limnische Fazies, aber in jedem Fall eine deutlich energiereichere Dynamik als während des Alleröd und Holozäns.

Auch Starkel (1995) registriert für die Weichsel einen Wechsel von organischen zu minerogenen Sedimenten beim Übergang vom Alleröd zur Dryas-III. Während der Dryas-III besteht eine deutliche Tendenz zur Sedimentation und lateralen Erosion im Weichseltal. Das Dryas-III-zeitliche Flussbett hat 3-4 m über dem heutigen Flussbettniveau gelegen.

Die Dryas-III zeigt nach VANDENBerGHe (1995) im europäischen Vergleich eine größere räumliche Variabilität des Prozessgeschehens als die vorangegangenen Zeitabschnitte. In einigen Talabschnitten geht die Anhäufung von Sediment weiter, in anderen ist Seitenerosion oder Einschneiden zu Beginn der Dryas-III zu beobachten. VANDENBERGHE (1995) vermutet, dass die Abkühlung des Klimas zu einer verminderten Evapotranspiration und erhöhten Ausräumung geführt hat. Bis zu einem gewissen Grad verringerte sich ebenfalls die Pflanzenbedeckung der Böden, was eine zunehmende geomorphologische Instabilität zur Folge hatte (VANDENBERGHE, 1995).

Da entsprechend dem aktuellen Stand der Forschung (BJÖRCK, 1995) eine Beeinflussung der geomorphologischen Prozesse in den Tälern Vorpommerns durch einen hochliegenden Wasserspiegel im Ostseebecken während des späten Alleröd und der Dryas-III entgegen den Ansichten von JankE \& REINHARD (1968) zunächst ausgeschlossen werden muss, stellt sich die Frage nach den Ursachen für die beschriebenen Phänomene. Die fluviale Reliefformung wird bekanntermaßen durch das Verhältnis von Schleppkraft des fließenden Wassers, der Menge und Korngröße des über die Hangabtragung und andere Vorgänge in den Fluss gelangten Materials (Last) und der Widerstandsfähigkeit des Gesteins im Erosionsbereich des Flusses gesteuert (KuGLER et al., 1988). Bekannt ist ebenfalls, dass sowohl Schleppkraft als auch Last klimaabhängig sind. 
So beschreibt Rose (1995) das Phänomen der Abfolge von Erosion und Sedimentation während der Dryas-III als typischen Fall von ,lead and lag“. Zunächst führten die auf die Klimaänderung schnell reagierenden Abflussschwankungen zu einer Erosion im Tal (zunehmende Schleppkraft). Dagegen reagierte die Vegetation im Einzugsgebiet langsamer, wobei die geomorphologische Instabilität der Böden und Sedimente erst allmählich größer wurde, so dass im Endeffekt die Zunahme des bereitgestellten Sedimentes (Last) hinter dem Klimasignal ,hinterher hinkte“".

Die jüngere spätglaziale Talentwicklung in Vorpommern kann auf Grundlage der eigenen Befunde sowie oben diskutierten regionalen und überregionalen Literatur unabhängig von einer Transgression im Ostseebecken begründet werden. Dabei hätten mit Beginn der spätglazialen Erwärmung und nach einer erosionsbasisbedingten Ausräumungsphase (JANKE \& REINHARDT, 1968 und JANKE, 1978 ) die zunehmend dichtere, im Alleröd schließlich waldartige Vegetation (DE KLERK et al., im Druck; DE KLERK, im Druck) sowie die wärmeren Winter zu einem ausgelicheneren Abflussregime und einem abnehmenden Sedimentanfall (Last) im Verlaufe der spätglazialen Warmphase geführt, was sich im Alleröd schließlich lithologisch durch einen deutlich geringeren minerogenen Anteil und die Tendenz zur Versumpfung bemerkbar macht. Nach einer möglichen Erosionsphase zu Beginn der Dryas-III (Rose, 1995; eigene Befunde) folgte schließlich wiederum eine Phase der minerogenen Akkumulation und Seitenerosion, da die Abflüsse nun jahreszeitlich gebündelt auftraten, gleichzeitig aber (bzw. mit zeitlicher Verzögerung) auch die Last zunahm (allmähliche Auflichtung der Vegetationsdecke; vgl. DE KLERK et al., im Druck; DE KLERK, im Druck).

\section{Danksagung}

Wir danken folgenden Mitarbeitern des Geographischen Institutes der Universität Greifswald: Prof. Dr. K. BILLwITz für sein Engagement als Leiter der AG Landschaftsgeschichte, Frau Brigitta LinTZEN und Frau Petra Wiese für die digitale Bearbeitung der Abbildungen, Frau Hannelore RABE für die Aufbereitung der Pollenproben sowie Herrn Dr. Knut Kaiser, Herrn Prof. Dr. Wolfgang JANKE sowie den Gutachtern für die Durchsicht des Manuskriptes.

\section{Schriftenverzeichnis}

AG Boden (1994): Bodenkundliche Kartieranleitung. - 4. Aufl.: 392 S.; Hannover (Schweizbart).

Barsch, H. \& Billwitz, K. [Hrsg.] (1990): Physisch Geographische Arbeitsmethoden. - 256 S.; Gotha (Haack).

Billwitz, K., De Klerk, P., Helbig, H., Kaiser, K. \& TER-BERger, T. (1998): Chronostratigraphie, geoökologische Entwicklung und menschliche Besiedlung vom Spätglazial zum Holozän in Nordostdeutschland. - Projektbericht zum DFG-Schwerpunktprogramm ,Wandel der Geobiosphäre seit den letzten 15.000 Jahren“, Universität Greifswald.

Billuwitz, K., Helbig, H., Kaiser, K., De Klerk, P., KüHn, P. \& Terberger, T. (2000): Untersuchungen zur spätpleistozänen bis frühholozänen Landschafts- und Besiedlungsgeschichte in Mecklenburg-Vorpommern. - Neubrandenburger Geologische Beiträge, 1: 24-38; Neubrandenburg (Geowissenschaftlicher Verein Neubrandenburg e.V.).

BJÖRCK, S. (1995): A Review of the history of the Baltic Sea, 13.0-8.0 ka BP. - In: ANDrÉn, T., BrunNBerG, L. \& Miller, U. [Hrsg.]: Proceedings from the Baltic Ice Lake-Yoldia Sea symposium, march 1993. - Quaternary International, 27: 19-41; Oxford (Pergamon).

Bock, W., Menke, B., Strehl, E. \& Ziemus, H. (1985): Neuere Funde des Weichselspätglazials in Schleswig-Holstein. - Eiszeitalter und Gegenwart, 35: 161-180; Öhringen (Schweizbart).

Dvareckas, V. (1995): The development of Lithuanian river in the Lateglacial and Holocene. - In: FRENZEL, B. [Hrsg.] (1995): European river activity and climatic change during the Lateglacial and early Holocene. - Paläoklimaforschung, 14, Special Issue: ESF Project „European Palaeoclimate and Man“: 113-121; Jena (Fischer).

FÆGRI, K. (1993): Bestimmungsschlüssel für die nordwesteuropäische Pollenflora. - Auszug aus F匞I, K. \& IVERSEN, J. (1989), übersetzt von E. LANGe: 85 S.; Jena (Fischer).

FæGri, K. \& Iversen, J. (1989): Textbook of Pollen analysis. - 4th edition (revised by FÆGRI, K., KALAND, P.E. \& KRZYwinSKI, K.): 328 S.; Chichester (Wiley).

FISCHER, U. (1995): Das NSG „Peenewiesen bei Gützkow" - Zur Vegetationsentwicklung im mittleren Peene-Talmoor. - unveröff. Diplomarbeit, Botanisches Institut Universität Greifswald: 125 S.; Greifswald.

HalliK, R. (1944): Pollenanalytische Untersuchungen im Postglazial Westpommerns. - Neues Jahrbuch für Mineralogie, Geologie und Paläontologie, 88: 40-84; Stuttgart.

Helbig, H. (1999-a): Die spätglaziale und holozäne Überprägung der Grundmoränenplatten in Vorpommern. - Greifswalder Geographische Arbeiten, 17: 110 S.; Greifswald.

— (1999-b): Die periglaziäre Überprägung der Grundmoränenplatten in Vorpommern. - Petermanns 
Geogr. Mitt., 143 (5+6): 373-386; Gotha (KlettPerthes).

HeLKe, U.-M. (1999): Das Revitalisierungspotential des Polders „Randow-Rustow"/Peenetal. - Unveröff. Diplomarbeit, Botanisches Institut Universität Greifswald: $115 \mathrm{~S}$.

Hoek, W.Z. (1997): Palaeogeography of Lateglacial vegetations. Aspects of Lateglacial and Early Holocene vegetation, abiotic landscape and climate in the Netherlands. - Nederlandse Geografische Studies, 230: 147 S.; Utrecht (Koninklijk Nederlands Aardrijkskundig Genootschap).

Hurtig, T. (1957): Physische Geographie von Mecklenburg. - 252 S.; Berlin.

JANKE, W. (1978): Schema der spät- und postglazialen Entwicklung der Talungen der spätglazialen Haffstauseeabflüsse. - Wissenschaftliche Zeitschrift der Universität Greifswald, 27 (1/2): 39-43; Greifswald.

JANKE, W. (1996): Landschaftsentwicklung und Formenschatz Mecklenburg-Vorpommerns seit der Weichsel-Eiszeit. - Zeitschrift für den Erdkundeunterricht, 12: 495-505; Berlin (Deutscher Verlag der Wissenschaften).

JANKE, W. \& ReINHARI, H. (1968): Zur spätglazialen Gletscherdynamik und Entwicklungsgeschichte der großen Talungen im Nordosten Mecklenburgs. Wissenschaftliche Zeitschrift der Universität Greifswald, 17 (1/2): 1-20; Greifswald.

JANSSEN, C.R. (1966): Recent pollen spectra from the deciduous and coniferous-deciduous forest of northeastern Minnesota: a study in pollen dispersal. - Ecology, 47: 804-825; Durham.

KAISER, K. \& JANKE, ,W. (1998): Bodenkundlich-geomorphologische und paläobotanische Untersuchungen im Ryckbecken bei Greifswald. - Bodendenkmalpflege in Mecklenburg-Vorpommern, 45, Jahrbuch 1997: 69-102; Lübstorf.

KaISER, K. \& Terberger, T. (1996): Archäologischgeowissenschaftliche Untersuchungen am spätpaläolithischen Fundplatz Nienhagen, Lkr. Nordvorpommern. - Bodendenkmalpflege in MecklenburgVorpommern, 43, Jahrbuch 1995: 7-48; Lübstorf.

Kaiser, K., De Klerk, P. \& Terberger, T. (1999): Die „Riesenhirschfundstelle“ von Endingen: geowissenschaftliche und archäologische Untersuchungen an einem spätglazialen Fundplatz in Vorpommern. - Eiszeitalter und Gegenwart, 49: 102-123; Hannover (Schweizbart).

De KLeRK, P. (im Druck): Changing vegetation patterns in the Endinger Bruch area (Vorpommern, NE Germany) during the Weichselian Lateglacial and Early Holocene. - Review of Palaeobotany and Palynology; Amsterdam (Elsevier).

De Klerk, P., Helbig, H., Helms, S., Janke, W., KrüGel, K., Kühn, P., Michaelis, D. \& Stolze, S. (im Druck): The Reinberg researches: palaeoecological and geomorphological studies of a kettle hole in Vorpommern (NE Germany), with special emphasis on a local vegetation during the Weichselian Pleniglacial/Lateglacial transition. - Greifswalder Geographische Arbeiten; Greifswald.
Kraus, M. (1999): Paläoökologische Untersuchungen im Recknitztal bei Bad Sülze (Kreis Nordvorpommern). - Unveröff. Diplomarbeit, Botanisches Institut der Universität Greifswald: $71 \mathrm{~S}$.

Kugler, H., Schwab, M. \& Billwitz, K. (1988): Allgemeine Geologie, Geomorphologie und Bodengeographie. - 223 S.; Gotha (Haack).

LitT, T. \& Stebich, M. (1999): Bio- and chronostratigraphy of the lateglacial in the Eifel region, Germany.- Quaternary International, 61: 5-16; Oxford (Pergamon).

LOWE, J.J. \& GRAY, J.M. (1980): The stratigraphic subdivision of the Lateglacial of NW Europe: a discussion. - In: LOWE, J.J., Gray, J.M. \& ROBINSON, J.E. [Hrsg.]: Studies in the lateglacial of North-west Europe. Including papers presented at a symposium of the Quaternary Research Association held at University College London, January 1979: $157-$ 175; Oxford (Pergamon).

Lowe, J.J. \& NASP Members (1995): Palaeoclimate of the North Atlantic seaboards during the last glacial/interglacial transition.- Quaternary International, 28: 51-61; Oxford (Pergamon).

Merkt, J. \& Müller, H. (1999): Varve chronology and palynology of the Lateglacial in Northwest Germany from lacustrine sediments of Hämelsee in Lower Saxony. - Quaternary International, 61: 4159; Oxford (Pergamon).

Michaelis, D. (2000): Die spät- und nacheiszeitliche Entwicklung der natürlichen Vegetation von Durchströmungsmooren in Mecklenburg-Vorpommern am Beispiel der Recknitz. - unveröff. Dissertation, Botanisches Institut der Universität Greifswald: $124 \mathrm{~S}$.

Moore, P.D., WebB, J.A. \& Collinson, M.E. (1991): Pollen analysis. - 216 S.; Oxford (Blackwell).

Punt, W. \& Blackmore, S. (1991): The northwest European pollen flora. VI. - 275 S.; Amsterdam (Elsevier).

Raukas, A. \& Mindel, A. (1995): Fluvial activity in Estonia in the Lateglacial and early Holocene. - In: FrenzeI, B. [Hrsg.] (1995): European river activity and climatic change during the Lateglacial and early Holocene. Paläoklimaforschung, 14, Special Issue: ESF Project „European Palaeoclimate and Man“: 101-112; Jena (Fischer).

Reinhard, H. (1963): Beitrag zur Entwicklungsgeschichte des Grenztales (NE-Mecklenburg) und seine Beziehung zur Litorina-Transgression. - Geologie, 12: 94-117; Berlin (Akademie-Verlag).

Rose, J. (1995): Lateglacial and early Holocene river activity in lowland Britain. - In: FrenzeL, B. [Hrsg.] (1995): European river activity and climatic change during the Lateglacial and early Holocene. - Paläoklimaforschung, 14, Special Issue: ESF Project „European Palaeoclimate and Man“: 51-74; Jena (Fischer).

RÜHBERG, N (1987): Die Grundmoräne des jüngsten Weichselvorstoßes im Gebiet der DDR.- Zeitschrift für Geologische Wissenschaften, 15 (6): 759-967; Berlin (Akademie-Verlag). 
SCHIRMER, U. (1999): Pollenstratigraphische Gliederung des Spätglazials im Rheinland. - Eiszeitalter und Gegenwart, 49: 132-143; Hannover (Schweizbart). STARKEL, L. (1995): The place of the Vistula river valley in the late Vistulian - early Holocene evolution of the European valleys. - In: Frenzel, B. [Hrsg.] (1995): European river activity and climatic change during the Lateglacial and early Holocene. Paläoklimaforschung, 14, Special Issue: ESF Project „European Palaeoclimate and Man“: 75-88; Jena (Fischer).

Usinger, H. (1985): Pollenstratigraphische, vegetations- und klimageschichtliche Gliederung des „Bölling-Alleröd Komplexes“ in Schleswig-Holstein und ihre Bedeutung für die Spätglazial-Stratigraphie in benachbarten Gebieten. - Flora, 177: 1-43; Jena (Fischer).

Usinger, H. (1998): Pollenanalytische Datierung spät-paläolitischer Fundschichten bei Ahrenshöft, Kr. Nordfriesland. Archäologische Nachrichten aus Schleswig-Holstein. - Mitteilungen der Archäologischen Gesellschaft Schleswig-Holstein e.V. und des Archäologischen Landesamtes SchleswigHolstein, 8: 50-73; Kiel (Archäologische Gesellschaft Schleswig-Holstein e.V., Archäologisches Landesamt Schleswig-Holstein).

VANDENBERGHe, J. (1995): Postglacial river activity and climate: state of the art and future prospects. - In: FrenzEL, B. [Hrsg.] (1995): European river activity and climatic change during the Lateglacial and early Holocene. - Paläo-klimaforschung, 14, Special Issue: ESF Project „European Palaeoclimate and Man": 1-10; Jena (Fischer).

Walker, M.J.C. (1995): Climatic changes in Europe during the last glacial/interstadial transition. Quaternary International, 28: 63-76; Oxford (Pergamon).

Werth, E. \& BaAs, J. (1936): Pollenanalytische Untersuchungen zur Vegetations- und Kulturgeschichte im deutschen Küstenbereich der Ostsee und Nordsee. - Abh. senckenberg. naturf. Ges., 34: 1-41: Frankfurt a. M. (Kramer). 